\title{
Study of Powder Density, Ag:Superconductor Ratio, and Microhardness of BSCCO-2212 Ag-Sheathed Wires and Tapes During Wire Drawing and Rolling
}

\author{
M. Karuna, Jeffrey A. Parrell, and David C. Larbalestier \\ Applied Superconductivity Center and Materials Science Program, University Of Wisconsin-Madison, USA
}

\begin{abstract}
Densification of $\mathrm{Bi}_{2} \mathrm{Sr}_{2} \mathrm{CaCu}_{2} \mathrm{O}_{x}$ (BSCCO-2212) powder cores in Ag-clad wires and tapes has been studied as a function of strain and initial packing density. Density was measured directly by etching away the silver and weighing the remaining core. $A$ universal feature in both wires and tapes was the attainment of maximum densities $(\sim 80 \%)$ at intermediate strains of $\sim 4$. The linear correlation between microhardness and density found for round wires breaks down for rolled tapes, apparently because the core separates into dense blocks separated by cracks.
\end{abstract}

\section{INTRODUCTION}

High temperature superconductor (HTS) composites are made by an unusual oxide-powder-in-tube (OPIT) method which has many differences from traditional superconductor fabrication methods. Among the requirements of any conductor fabrication process are the need to understand the deformation mechanisms of the composite, to predict the final filament size and shape and to control the superconductor to normal metal ratio. So far, little information on these points for HTS composites has been publicly presented. An important question concerns control of the Ag:BSCCO ratio. The normal metal:superconductor ratio is almost invariably larger (typically $>3: 1$, rather than $<2: 1)$ than in low temperature superconductor composites, not as a matter of explicit design but rather because the deformation characteristics are not yet well enough understood to permit lower ratios to be reliably made. Good conductor design would permit the $\mathrm{Ag}$ :BSCCO ratio to be predicted at the design stage but since an OPIT composite cannot be packed at full density, this requires a knowledge of the powder densification and deformation behavior during wire drawing and tape rolling.

This question has other important facets. One is the need to minimize sausaging and other irregularities of the filament structure. BSCCO filaments easily develop irregularities (sausaging) which can exert a very deleterious effect on the transport critical current density $\left(J_{c}\right)[1,2]$. Some of the sausaging is provoked by the powder properties, principally its relative packing density, size, shape and phase distribution, and some by the billet design and deformation schedule. What role the initial powder density

Manuscript received October 16, 1994.

This work was supported by ARPA (N00014 J-4115) and EPRI (RP 8009-05). at billet packing $\left(\rho_{\mathrm{ro}}\right)$ plays is unclear but, since the relative powder density $\left(\rho_{r}\right)$ of the core should affect the strength of the core, it is widely thought that relative density is an important parameter [3]. A second issue also influences the $\mathrm{J}_{\mathrm{c}^{\circ}}$. This is the role that the relative density of the deformed core plays in the electrical connectivity of the BSCCO core. Cracks, voids or other significant variations of the powder density must be expected in a low density core and must also be expected to degrade the transport $\mathrm{J}_{\mathrm{c}}$ of the conductor.

Yamada et al. [4] estimated the density of unreacted cores in BSCCO-2223 Ag-sheathed tapes based on a linear correlation between hardness and density in bulk reacted BSCCO-2212 samples. They reported [5] that $\rho$, increased with increasing strain in both wires and in tapes and that the attainment of high density $(\sim 95 \%)$ correlated with very high $\mathbf{J}_{c}$ values. The correlation between the reacted hardness of BSCCO-2223 tapes and their $\mathbf{J}_{\mathrm{c}}$ was established by Parrell, Dorris, and Larbalestier [6,7]. However, most density information is inferred, rather than measured and the lack of hard data of BSCCO cores was the reason for this study. We wanted to understand what the consequences of varying the initial packing density of powder in a BSCCO-2212 Agclad tape were. Here we present data on the densification, on the apparent Ag:BSCCO ratio, and on the hardness as a function of drawing strain. We find that "loose packed" billets densify very quickly and soon reach the same density as billets containing an isostatically pressed core. Microhardness measurements can be correlated to the density in drawn wires but the correlation breaks down in rolled tapes.

\section{EXPERIMENTAL PROCEDURE}

Two commercially pure Ag tubes having outer and inner diameters of 14.2 and $7.2 \mathrm{~mm}$ respectively ( $\mathrm{Ag}$ :Hole ratio of 2.8:1) were packed with the same commercially produced BSCCO-2212 powder [8]. One tube was filled by pouring in powder and periodically tapping it so as to produce a loosely packed powder with $\rho_{\text {ro }} \sim 23 \%$ of theoretical $\left(6.6 \mathrm{~g} / \mathrm{cm}^{3}\right)$. The other tube was filled with a slug which had been cold isostatically pressed (CIP'ed) at $350 \mathrm{MPa}$ having $\rho_{\mathrm{ro}} \sim 72 \%$ of theoretical. The billets were subsequently processed identically, being cold drawn from $14.2 \mathrm{~mm}$ dia. with $10 \%$ area reduction per pass to a diameter of $1.8 \mathrm{~mm}$ using standard dies with a half angle of $6^{\circ}$. The wires were then rolled flat with $5 \%$ thickness reduction per pass to a 
thickness of $0.1 \mathrm{~mm}$. A piece of the loose packed billet was also drawn round from 1.8 to $0.51 \mathrm{~mm}$ dia.

Density was determined from short samples which were removed from the back of the composites at regular intervals of strain during the deformation process. The samples were cut using a diamond saw and the ends were squared by grinding. The Ag cladding was removed by etching in a 2.5:1 solution of $\mathrm{NH}_{4} \mathrm{OH}$ and $\mathrm{H}_{2} \mathrm{O}_{2}$ (30 vol.\%) and the remaining 2212 powder core was weighed. At least two transverse cross sections corresponding to each sampled strain were mounted for metallography. The areas of the 2212 core and the $\mathrm{Ag}$ sheath were measured on polished cross sections by image analysis. The density was then calculated by dividing the core mass by the volume, determined from the product of the transverse cross-section and the sample length. Since the quantity of $\mathrm{Ag}$ was large in the early stages of wire drawing, it was not etched away from large diameter samples. The mass of 2212 cores in the first three samples was instead estimated by subtracting the weight of the silver (calculated using $10.6 \mathrm{~g} / \mathrm{cm}^{3}$ for the density of $\mathrm{Ag}$ ) from the overall weight of the sample.

To confirm that BSCCO-2212 does not react with the above etch, a $1 \mathrm{~cm}$ piece of the CIP'ed slug was dropped in the etch for 30 minutes and then re-weighed. The weight increased by only $1 \%$. One of the etched cores was also maintained at $200^{\circ} \mathrm{C}$ in an atmosphere of $\mathrm{N}_{2}$ for five hours, simulating a very exaggerated drying cycle. No perceptible weight change was detected. We concluded that our etching and drying procedures did not adversely affect the core.

Vickers microhardness was measured on polished transverse cross sections ( $10 \mathrm{~g}$ load, $15 \mathrm{~s}$ load time). Equal numbers of indentations were made at the center and edge of each core. Between 5-10 indentations were made on each sample and the average and standard deviations calculated We found that the surface polish had to be extremely uniform for a valid comparison of $\mathbf{H}_{v}$ between samples to be made and that the tests had to be performed immediately after polishing. As in reference [4], we found that $\mathrm{H}_{v}$ was higher towards the wire and tape center.

\section{RESULTS}

Fig. 1 shows that the BSCCO-2212 powder consisted of partially sintered agglomerates $(<50 \mu \mathrm{m})$ of smaller $(<5$ $\mu \mathrm{m})$ particles when at the stage of billet packing. This view of the powder may usefully be contrasted with that discussed later in Fig. 5.

Fig. 2 contains plots of $\rho_{r}$ versus true deformation strain ( $\varepsilon$ $=\ln \left(\mathrm{a}_{\mathrm{f}} / \mathrm{a}_{\mathrm{t}}\right)$ where $\mathrm{a}_{\mathrm{t}}=$ initial area at packing and $\mathrm{a}_{\mathrm{f}}=$ area of measurement). Because the cores never fully densified, the strain calculated from the external dimensions has some uncertainty associated with changes in the powder core density but it still serves as a good operational fabrication parameter. For the loose packed powder, the density started at $23 \%$ and rose rapidly to $70 \%$ within a strain of 1.5 . The
CIP'ed core density started at $72 \%$ and increased only slightly to about $75-80 \%$. after a strain of $\sim 4$. Thereafter the density of both tapes decreased slowly during rolling. Furthermore, the density of the loose packed wire which was drawn from a strain of 4.0 to 6.6 also decreased. The maximum density attained was not a function of the initial packing density or the $\mathrm{Ag}:$ Hole ratio.

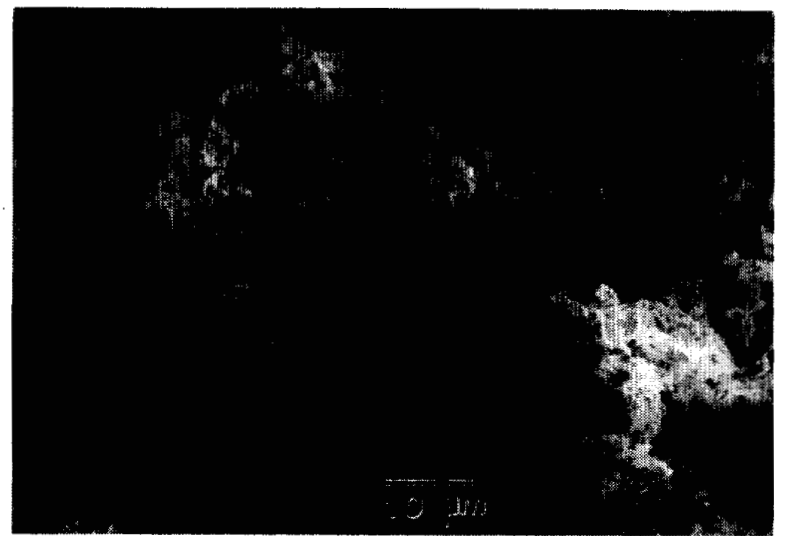

Fig.1 SEM micrograph of the precursor powder. The powder consisted of large agglomerates of particles having a diameter of order $50 \mu \mathrm{m}$.

Fig. 3 shows the Ag:Hole ratio as a function of strain. The ratio was almost independent of strain for the CIP'ed composite, remaining in the range 2.8-3.5:1 at all strains. By contrast, the $\mathrm{Ag}$ :Hole ratio of the loose packed billet started at 2.8:1 but then increased to more than 10:1. The error bars in Figs. 2a, 2b and 3 correspond to the maximum and minimum values of the measured areas.

Fig. 4 shows the Vickers hardness $\left(\mathrm{H}_{v}\right)$ as a function of strain. The error bars correspond to one standard deviation on each side of the mean. The hardness varied from $\sim 70$ to $140 \mathrm{~kg} / \mathrm{mm}^{2}$ for the loose packed billet and from $\sim 90$ to 130 $\mathrm{kg} / \mathrm{mm}^{2}$ for the CIP'ed billet. The hardness values also showed a peak similar to the density peak as a function of strain. However, the two peaks do not coincide at exactly the same strain for the CIP'ed material.

Figure 5 shows the exposed broad surface of the CIP'ed tape at a strain of $\sim 6$ at a point where the relative density has diminished somewhat from its maximum. The surface exhibits a marked striping which runs perpendicular to the rolling direction. Inset is a higher magnification view of the surface which appears to indicate that the powder particles are more regular in shape and size $(\sim 1-2 \mu \mathrm{m}$ in diameter) than when they were loaded into the billet. We interpret the stripes as surface ridges which are associated with transverse cracks of the powder core.

We measured the core density of a fully processed tape and found that it increased by from $\sim 75 \%$ to $\sim 85 \%$ upon heat treatment, based on the same ideal density for the core. The cross-sectional area of the core remained the same 
before and after heat-treatment, thus suggesting that the processed tapes contain $\sim 15 \%$ of distributed void space.
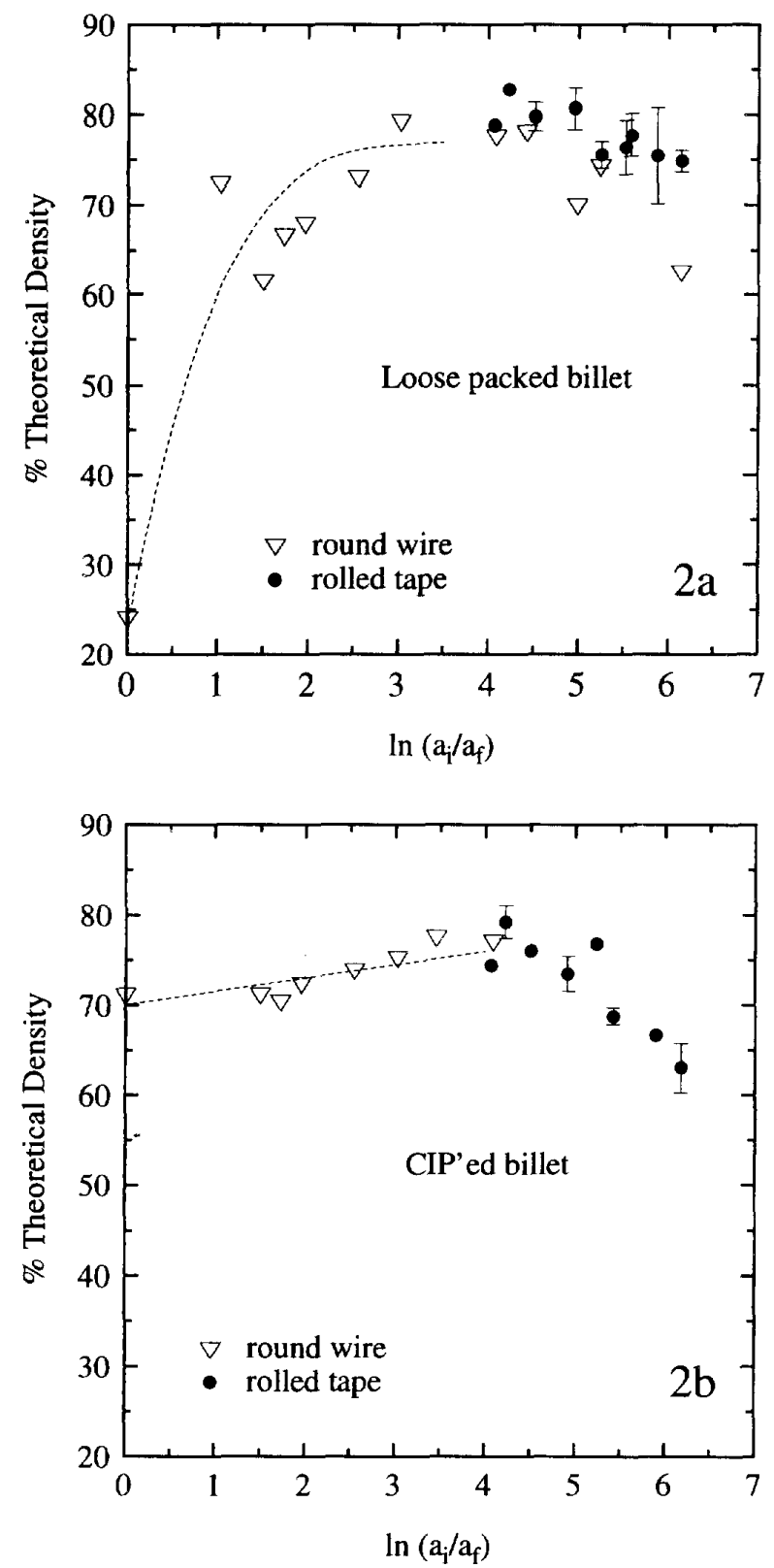

Figs $2 \mathrm{a}$ and $2 \mathrm{~b}$. Plots of $\%$ theoretical density as a function of true strain for the loose packed and CIP'ed billets, respectively. Note the large difference in the initial packing density, and that both composites reach a maximum density of about $75 \%$ of theoretical at a strain of $\sim 4$. Lines are drawn as a guide to the eye.

\section{DisCussion}

A surprising feature of both wires and tapes is the attainment of maximum density at an intermediate strain of $\sim 4$. In both composites, the maximum density was around $80 \%$. Such a value is close to the theoretical maximum packing of equal size spheres, thus suggesting that the principal deformation method is one of hard particle sliding and agglomerate fracture. This is consistent with the approximately spherical shape of the particles in Fig. 5. The present observed decrease in density might have several causes. Some slip is possible in both BSCCO-2212 and 2223 but it seems most reasonable to suppose that particle sliding and fracture are the dominant deformation mechanisms. The smaller size and more nearly spherical shape of the powder after a large strain is direct evidence for this (compare Figs. 1 and 5). The ridges in Fig. 5 appear to represent the intersection of shear bands with the surface. Based on this we interpret the decline in density of tapes at higher strains as being the consequence of the core segregating into dense blocks separated by actual or incipient cracks. The relative amount of the core in contact with the silver sheath increases with increasing strain and since the edges have lower hardness compared to the center the overall density decreases. Moreover, with increasing deformation, the interface gets progressively rougher leading to higher frictional forces which might also lead to a decrease in density. Hardness data show a nearly linear relationship with density for the wires. However the correlation breaks down for the tapes most probably due to the formation of blocks within the core.

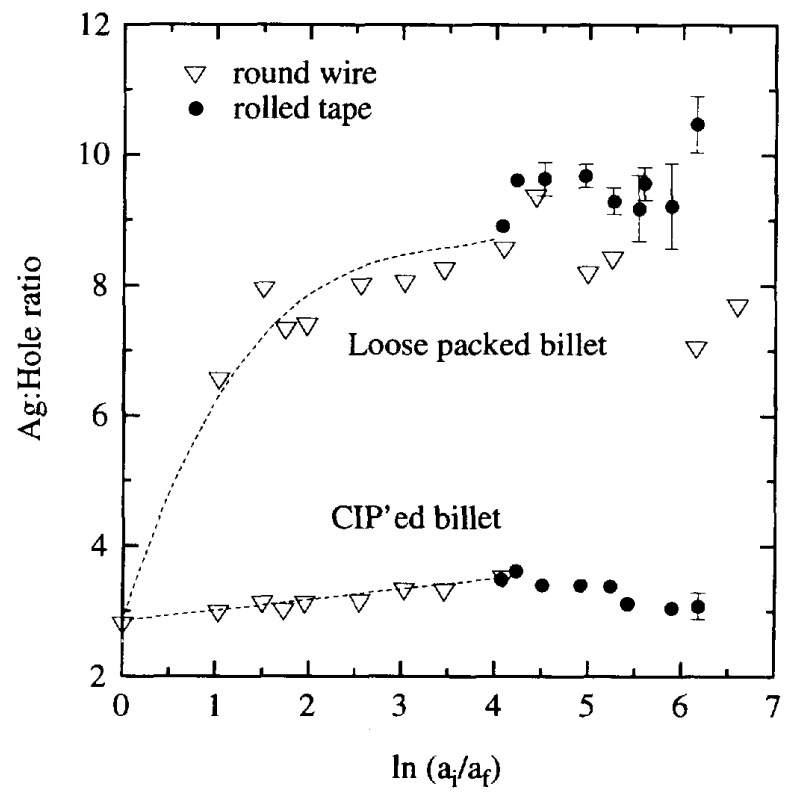

Fig. 3. Ag:Hole ratio as a function of true strain. Note that the ratio for the loose packed billet increases drastically, while the ratio on the CIP'ed billet changes much less. Lines are drawn as a guide to the eye. 

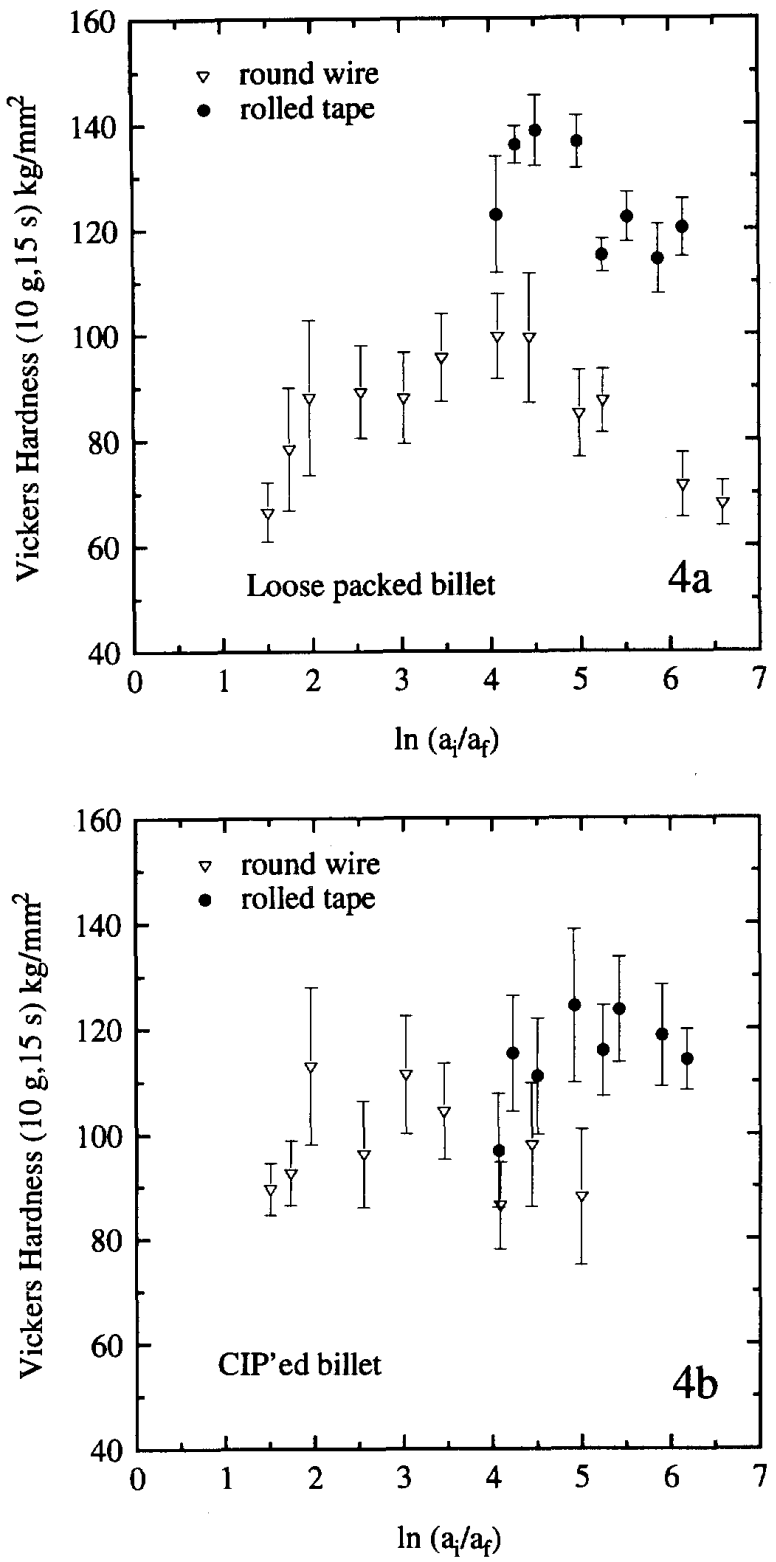

Figs. 4a and $4 b$. Plot of $H_{v}$ as a function of true strain for the loose packed and CIP'ed composites, respectively

\section{CONCLUSIONS}

We have directly measured the density of the powder core as a function of strain for two BSCCO-2212 composites. The maximum density of $\sim 80 \%$ was achieved at intermediate strains ( $\sim 4)$ and was not a function of the initial packing density or the $\mathrm{Ag}$ :BSCCO ratio. The Vickers hardness exhibits an increase with increasing deformation strain but there is not an exact correlation with the density data, although they showed a similar trend with strain.
Separation of the core into a cracked array of denser blocks separated by cracks is believed to be the reason why the direct correlation between hardness and density breaks down in tapes.

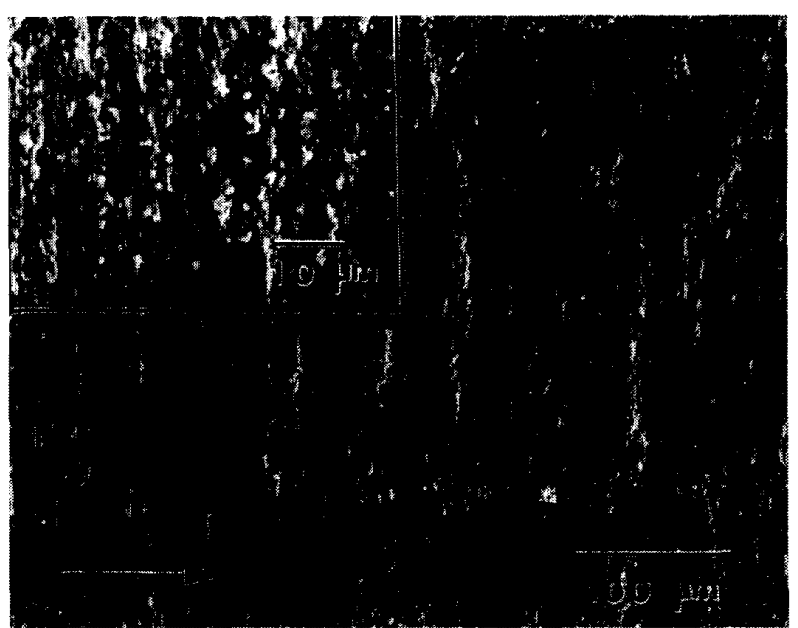

Fig. 5. SEM micrograph of the broad exposed face of the BSCCO core from a sample of the CIP'ed billet at a strain of $\sim 6$ (Inset: Same sample at higher magnification). The rolling direction is indicated by the arrow. The Ag was removed by etching.

\section{ACKNOWLEDGMENTS}

We would like to acknowledge the experimental assistance of W.S. Starch, and general discussions of the deformation properties of powders with P.D. Jablonski, T.C. Willis and R.W. Heussner (UW), with R. Asaro and S. Schoenfeld (UC-San Diego), Q. Li (American Superconductor Corp.), J. Kajuch (NCEMT) and V. Sikka (Oak Ridge National Laboratory).

\section{REFERENCES}

[1] K. Osamura, S.S. Oh, and S. Ochiai, Supercond. Sci. Technol. 5 (1992) 1-6.

[2] H.S. Edelman and D.C. Larbalestier, Adv. Cryo. Engr 40 (1994) 749 754.

[3] R. Asaro, private communication.

[4] Y. Yamada, M. Satou, S. Murase, T. Kitamura and Y. Kamisada, Proc. of ISS'92, (Springer,Tokyo,1993) p. 717.

[5] M. Satou, Y. Yamada, S. Murase, T. Kitamura and Y. Kamisada, Appl. Phys. Lett. 64 (1994) 640-644.

[6] J.A. Parrell, S.E. Dorris, and D.C. Larbalestier, Adv. Cryo. Engr 40 (1994) 193-200.

[7] J.A. Parrell, S.E. Dorris, and D.C. Larbalestier, Physica C. 231 (1994) 137-146.

[8] The 2212 powder was obtained from Seattle Specialty Ceramics. 